\title{
Oscillation criteria for first-order dynamic equations with nonmonotone delays
}

\author{
Nurten Kılıç ${ }^{1}$ (D), Özkan Öcalan*2 (D) \\ ${ }^{1}$ Kütahya Dumlupınar University, Faculty of Science and Arts, Department of Mathematics, 43100, \\ Kütahya, Turkey \\ ${ }^{2}$ Akdeniz University, Faculty of Science, Department of Mathematics, 07058 Antalya, Turkey
}

\begin{abstract}
In this paper, we consider the first-order dynamic equation as the following:

$$
x^{\Delta}(t)+\sum_{i=1}^{m} p_{i}(t) x\left(\tau_{i}(t)\right)=0, t \in\left[t_{0}, \infty\right)_{\mathbb{T}}
$$

where $p_{i} \in C_{r d}\left(\left[t_{0}, \infty\right)_{\mathbb{T}}, \mathbb{R}^{+}\right), \tau_{i} \in C_{r d}\left(\left[t_{0}, \infty\right)_{\mathbb{T}}, \mathbb{T}\right)(i=1,2, \ldots, m)$ and $\tau_{i}(t) \leq$ $t$, $\lim _{t \rightarrow \infty} \tau_{i}(t)=\infty$. When the delay terms $\tau_{i}(t)(i=1,2, \ldots, m)$ are not necessarily monotone, we present new sufficient conditions for the oscillation of first-order delay dynamic equations on time scales.
\end{abstract}

Mathematics Subject Classification (2020). 34C10, 34N05, 39A12, 39A21

Keywords. dynamic equations, nonmonotone delay, oscillation, time scales

\section{Introduction}

As is well known, after Stefan Hilger [15], [16] introduced the theory of dynamic equations on time scales (or measure chain) in his Ph.D. thesis in 1988, a lot of papers have been devoted to this subject field. Especially, the oscillatory behaviour of solutions of differential/difference and dynamic equations has been studied by many authors. See, for example, [1-34] and the references cited therein. Consider the first-order delay dynamic equation

$$
x^{\Delta}(t)+\sum_{i=1}^{m} p_{i}(t) x\left(\tau_{i}(t)\right)=0, \quad t \in\left[t_{0}, \infty\right)_{\mathbb{T}},
$$

where $\mathbb{T}$ is a time scale unbounded above with $t_{0} \in \mathbb{T}, p_{i} \in C_{r d}\left(\left[t_{0}, \infty\right)_{\mathbb{T}}, \mathbb{R}_{0}^{+}\right), \tau_{i} \in$ $C_{r d}\left(\left[t_{0}, \infty\right)_{\mathbb{T}}, \mathbb{T}\right)(i=1,2, \ldots, m)$ are not necessarily monotone such that

$$
\tau_{i}(t) \leq t \text { for all } t \in \mathbb{T}, \quad \lim _{t \rightarrow \infty} \tau_{i}(t)=\infty .
$$

A function $p: \mathbb{T} \rightarrow \mathbb{R}$ is called positively regressive (we write $p \in \mathcal{R}^{+}$) if it is rd-continuous and satisfies $1+\mu(t) p(t)>0$ for all $t \in \mathbb{T}$, where $\mu: \mathbb{T} \rightarrow \mathbb{R}_{0}^{+}$is the graininess function defined by $\mu(t):=\sigma(t)-t$ with the forward jump operator $\sigma: \mathbb{T} \rightarrow \mathbb{T}$ defined by $\sigma(t)=$ $\inf \{s \in \mathbb{T}: s>t\}$ for $t \in \mathbb{T}$. A point $t \in \mathbb{T}$ is called right-dense if $\sigma(t)=t$ (or equivalently

*Corresponding Author.

Email addresses: nurten.kilic@dpu.edu.tr (N. Kılıç), ozkanocalan@akdeniz.edu.tr (Ö. Öcalan)

Received: 13.01.2020; Accepted: 10.06.2020 
$\mu(t)=0)$ holds, otherwise it is called right-scattered. The readers are referred to Bohner and Peterson [2] for further details concerning the time scales calculus.

A function $x: \mathbb{T} \rightarrow \mathbb{R}$ is called a solution of the equation (1.1), if $x(t)$ is delta differentiable for $t \in \mathbb{T}^{\kappa}$ and satisfies equation (1.1) for $t \in \mathbb{T}^{\kappa}$. We say that a solution $x$ of equation (1.1) has a generalized zero at $t$ if $x(t)=0$ or if $\mu(t)>0$ and $x(t) x(\sigma(t))<0$. Let $\sup \mathbb{T}=\infty$ and then a nontrivial solution $x$ of equation (1.1) is called oscillatory on $[t, \infty)$ if it has arbitrarily large generalized zeros in $[t, \infty)$.

For $m=1$, equation (1.1) reduces to

$$
x^{\Delta}(t)+p(t) x(\tau(t))=0, \quad t \in\left[t_{0}, \infty\right)_{\mathbb{T}} .
$$

Now, we give some well-known tests on oscillatory behaviour of (1.3). In 2002, Zhang and Deng [32], using the cylinder transforms, proved that if $\tau(t)$ is eventually nondecreasing and

$$
\limsup _{t \rightarrow \infty} \sup _{\lambda \in E}\left\{\lambda e_{-\lambda p}(t, \tau(t))\right\}<1
$$

where $E=\{\lambda: \lambda>0,1-\lambda p(t) \mu(t)>0\}$ and in 2005, Bohner [4], using exponential functions notation, proved that if $\tau(t)$ is eventually nondecreasing and

$$
\limsup _{t \rightarrow \infty} \sup _{-\lambda p \in \mathcal{R}^{+}}\left\{\lambda e_{-\lambda p}(t, \tau(t))\right\}<1,
$$

where

$$
e_{-\lambda p}(t, \tau(t))=\exp \left\{\int_{\tau(t)}^{t} \xi_{\mu(s)}(-\lambda p(s)) \Delta s\right\}
$$

and

$$
\xi_{h}(z)=\left\{\begin{array}{ll}
\frac{\log (1+h z)}{h} & , \text { if } h \neq 0 \\
z & , \text { if } h=0
\end{array},\right.
$$

then all solutions of equation (1.3) are oscillatory.

In 2005, Zhang et al. [33] and in 2006, Şahiner and Stavroulakis [27], using different technique, obtained that if $\tau(t)$ is eventually nondecreasing and

$$
\limsup _{t \rightarrow \infty} \int_{\tau(t)}^{\sigma(t)} p(s) \Delta s>1
$$

then all solutions of equation (1.3) are oscillatory. In 2005, Zhang et al. [33] (See also Agarwal and Bohner [1, Theorem 1]) established the following result. Assume that $\tau(t)$ is eventually nondecreasing and

$$
m:=\liminf _{t \rightarrow \infty} \int_{\tau(t)}^{t} p(s) \Delta s>\frac{1}{e}
$$

then all solutions of (1.3) oscillate.

In 2006, Şahiner and Stavroulakis [27] found out that if $\tau(t)$ is eventually nondecreasing,

$$
\liminf _{t \rightarrow \infty} \int_{\tau(t)}^{t} p(s) \Delta s>c \text { and } \limsup _{t \rightarrow \infty} \int_{\tau(t)}^{t} p(s) \Delta s>1-\frac{c^{2}}{4},
$$

where $c \in(0,1)_{\mathbb{R}}$, then every solution of equation (1.3) oscillates. Furthermore, Agarwal and Bohner [1] improved the condition (1.6) as follows: 
If $\tau(t)$ is eventually nondecreasing,

$$
\liminf _{t \rightarrow \infty} \int_{\tau(t)}^{t} p(s) \Delta s>c \text { and } \limsup _{t \rightarrow \infty} \int_{\tau(t)}^{t} p(s) \Delta s>1-(1-\sqrt{1-c})^{2}
$$

where $c \in(0,1)_{\mathbb{R}}$, then every solution of equation (1.3) oscillates.

Also, in 2016, Karpuz and Öcalan [19] enhanced the condition (1.7) by extending the second integral condition to the larger interval $[\tau(t), t]_{\mathbb{T}}$ as the following:

Assume that $\tau(t)$ is eventually nondecreasing and

$$
\liminf _{t \rightarrow \infty} \int_{\tau(t)}^{t} p(s) \Delta s>c \text { and } \limsup _{t \rightarrow \infty} \int_{\tau(t)}^{\sigma(t)} p(s) \Delta s>1-(1-\sqrt{1-c})^{2},
$$

where $c \in(0,1)_{\mathbb{R}}$. Then every solution of equation (1.3) oscillates.

Zhang et al. [33] established the following result. Assume that $\tau(t)$ is eventually nondecreasing and $m \in\left[0, \frac{1}{e}\right]$ (where $m$ is defined by (1.5)). Moreover, if

$$
\limsup _{t \rightarrow \infty} \int_{\tau(t)}^{\sigma(t)} p(s) \Delta s>\frac{1+\ln \lambda_{1}}{\lambda_{1}}-\frac{1-m-\sqrt{1-2 m-m^{2}}}{2},
$$

where $\lambda_{1} \in[1, e]$ is the unique root of the equation $\lambda=e^{m \lambda}$, then all solutions of equation (1.3) are oscillatory. It is obvious that, since

$$
\frac{1+\ln \lambda_{1}}{\lambda_{1}} \leq 1 \text { for } \lambda_{1} \in[1, e]
$$

the condition (1.9) implies

$$
\limsup _{t \rightarrow \infty} \int_{\tau(t)}^{\sigma(t)} p(s) \Delta s>1-\frac{1-m-\sqrt{1-2 m-m^{2}}}{2} .
$$

Clearly, when $0<c \leq \frac{1}{e}$, it is easy to verify that

$$
\frac{1-c-\sqrt{1-2 c-c^{2}}}{2}>(1-\sqrt{1-c})^{2}>\frac{c^{2}}{4}
$$

and therefore the condition (1.10) is weaker than the conditions (1.6) and (1.8).

Now, we assume that $\tau(t)$ is not necessarily monotone. Set

$$
h(t)=\sup _{s \leq t} \tau(s), \quad t \in \mathbb{T}, t \geq 0 .
$$

Clearly, $h(t)$ is nondecreasing and $\tau(t) \leq h(t)$ for all $t \geq 0$.

In 2017, Öcalan, Özkan and Yıldız [24, Theorem 2.2] studied the equation (1.3) when $\tau(t)$ is not necessarily monotone and obtained the following result.

Theorem A. If

$$
\limsup _{t \rightarrow \infty} \int_{h(t)}^{\sigma(t)} p(s) \Delta s>1
$$

where $h(t)$ is defined by (1.11), then every solution of (1.3) is oscillatory.

Finally, Öcalan [25, Corollary 2.4] established the following result when $\tau(t)$ is not necessarily monotone. 
Theorem B. If

$$
\liminf _{t \rightarrow \infty} \int_{h(t)}^{t} p(s) \Delta s=\liminf _{t \rightarrow \infty} \int_{\tau(t)}^{t} p(s) \Delta s>\frac{1}{e},
$$

where $h(t)$ is defined by (1.11), then all solutions of (1.3) oscillate.

A slight modification in the proofs of Theorems $A$ and $B$ leads to the following result.

Theorem 1.1. Assume that all the conditions of Theorems $A$ and $B$ hold. Then (i) the dynamic inequality

$$
x^{\Delta}(t)+p(t) x(\tau(t)) \leq 0, \quad t \in\left[t_{0}, \infty\right)_{\mathbb{T}}
$$

has no eventually positive solutions;

(ii) the dynamic inequality

$$
x^{\Delta}(t)+p(t) x(\tau(t)) \geq 0, \quad t \in\left[t_{0}, \infty\right)_{\mathbb{T}}
$$

has no eventually negative solutions.

\section{Main results}

In this section, we present some new sufficient conditions for the oscillation of all solutions of (1.1), under the assumption that the arguments $\tau_{i}(t)(i=1,2, \ldots, m)$ are not necessarily monotone. Set

$$
h_{i}(t)=\sup _{s \leq t}\left\{\tau_{i}(s)\right\} \text { and } h(t)=\max _{1 \leq i \leq m}\left\{h_{i}(t)\right\}, t \in \mathbb{T}, t \geq 0 .
$$

Clearly, $h_{i}(t)(i=1,2, \ldots, m)$ are nondecreasing and $\tau_{i}(t) \leq h_{i}(t) \leq h(t)(i=1,2, \ldots, m)$ for all $t \geq 0$.

The following lemma was given by Şahiner and Stavroulakis [27].

Lemma 2.1. Assume that $f: \mathbb{T} \rightarrow \mathbb{R}$ is rd-continuous, $g: \mathbb{T} \rightarrow \mathbb{R}$ is nonincreasing and $\tau: \mathbb{T} \rightarrow \mathbb{T}$ is nondecreasing. If $b<u$, then

$$
\int_{b}^{\sigma(u)} f(s) g(\tau(s)) \Delta s \geq g(\tau(u)) \int_{b}^{\sigma(u)} f(s) \Delta s .
$$

The following result is easily obtained by using the similar way in the proof of Lemma 2.3 in [24].

Lemma 2.2. Assume that (2.1) holds and $\alpha>0$. Then, we have

$$
\alpha:=\liminf _{t \rightarrow \infty} \int_{h(t)}^{t} \sum_{i=1}^{m} p_{i}(s) \Delta s=\liminf _{t \rightarrow \infty} \int_{\tau(t)}^{t} \sum_{i=1}^{m} p_{i}(s) \Delta s,
$$

where $\tau(t)=\max _{1 \leq i \leq m}\left\{\tau_{i}(t)\right\}, t \in \mathbb{T}, t \geq 0$.

Theorem 2.3. Assume that $-\sum_{i=1}^{m} p_{i} \in \mathcal{R}^{+}$. If $\tau_{i}(t)(i=1,2, \ldots, m)$ are not necessarily monotone and

$$
\limsup _{t \rightarrow \infty} \int_{h(t)}^{\sigma(t)} \sum_{i=1}^{m} p_{i}(s) \Delta s>1
$$

or

$$
\liminf _{t \rightarrow \infty} \int_{\tau(t)}^{t} \sum_{i=1}^{m} p_{i}(s) \Delta s>\frac{1}{e}
$$


where $h(t)$ is defined by (2.1) and $\tau(t)=\max _{1 \leq i \leq m}\left\{\tau_{i}(t)\right\}$. Then all solutions of (1.1) oscillate.

Proof. Assume, for the sake of contradiction, that there exists a nonoscillatory solution $x(t)$ of (1.1). Since $-x(t)$ is also a solution of (1.1), we can confine our discussion only to the case where the solution $x(t)$ is eventually positive. Then, there exists $t_{1}>t_{0}$ such that $x(t), x\left(\tau_{i}(t)\right)>0(i=1,2, \ldots, m)$, for all $t \geq t_{1}$. Thus, from (1.1) we have

$$
x^{\Delta}(t)=-\sum_{i=1}^{m} p_{i}(t) x\left(\tau_{i}(t)\right) \leq 0 \text { for all } t \geq t_{1},
$$

which means that $x(t)$ is an eventually nonincreasing function. In view of this and $\tau_{i}(t) \leq$ $\tau(t)(i=1,2, \ldots, m),(1.1)$ gives

$$
x^{\Delta}(t)+\left(\sum_{i=1}^{m} p_{i}(t)\right) x(\tau(t)) \leq 0, \quad t \geq t_{1} .
$$

Comparing (2.2) and (2.3), we obtain a contradiction to Theorem 1.1. Thus, the proof of the theorem is completed.

Now, we consider the case where $0<\alpha \leq \frac{1}{e}$. Then, we will obtain new oscillatory condition for all solutions of (1.1). We need the following lemma to establish our result. When the case $\tau_{i}(t)(i=1,2, \ldots, m)$ are not necessarily monotone, the following lemma can be easily obtained by using the similar process in [33, Lemma 2.4]. So, the proof of the following result is omitted here.

Lemma 2.4. Assume that $\tau_{i}(t)(i=1,2, \ldots, m)$ are not necessarily monotone. Let $0 \leq$ $\alpha \leq \frac{1}{e}$ and $x(t)$ be an eventually positive solution of Eq.(1.1). Then, we get

$$
\liminf _{t \rightarrow \infty} \frac{x(\sigma(t))}{x(h(t))} \geq \frac{1-\alpha-\sqrt{1-2 \alpha-\alpha^{2}}}{2},
$$

where $h(t)$ is defined by (2.1) and $\tau(t)=\max _{1 \leq i \leq m}\left\{\tau_{i}(t)\right\}$.

Theorem 2.5. Assume that $-\sum_{i=1}^{m} p_{i} \in \mathcal{R}^{+}$and $0 \leq \alpha \leq \frac{1}{e}$. If $\tau_{i}(t)(i=1,2, \ldots, m)$ are not necessarily monotone and

$$
\limsup _{t \rightarrow \infty} \int_{h(t)}^{\sigma(t)} \sum_{i=1}^{m} p_{i}(s) \Delta s>1-\frac{1-\alpha-\sqrt{1-2 \alpha-\alpha^{2}}}{2},
$$

where $h(t)$ is defined by (2.1). Then all solutions of (1.1) oscillate.

Proof. Assume, for the sake of contradiction, that there exists a nonoscillatory solution $x(t)$ of (1.1). Since $-x(t)$ is also a solution of (1.1), we can confine our discussion only to the case where the solution $x(t)$ is eventually positive. Then, there exists $t_{1}>t_{0}$ such that $x(t), x\left(\tau_{i}(t)\right)>0(i=1,2, \ldots, m)$, for all $t \geq t_{1}$. Thus, from (1.1) we have

$$
x^{\Delta}(t)=-\sum_{i=1}^{m} p_{i}(t) x\left(\tau_{i}(t)\right) \leq 0 \text { for all } t \geq t_{1},
$$

which means that $x(t)$ is an eventually nonincreasing function. In view of this and $\tau_{i}(t) \leq$ $h_{i}(t) \leq h(t)(i=1,2, \ldots, m)$, Eq.(1.1) gives

$$
x^{\Delta}(t)+\sum_{i=1}^{m} p_{i}(t) x(h(t)) \leq 0, \quad t \geq t_{1} .
$$


Integrating (2.6) from $h(t)$ to $\sigma(t)$ and taking into account the facts that the function $h(t)$ is nondecreasing and the function $x(t)$ is nonincreasing, we obtain

$$
x(\sigma(t))-x(h(t))+\int_{h(t)}^{\sigma(t)} \sum_{i=1}^{m} p_{i}(s) x(h(s)) \Delta s \leq 0 .
$$

Therefore, by using Lemma 2.1, we get

$$
x(\sigma(t))-x(h(t))+x(h(t)) \int_{h(t)}^{\sigma(t)} \sum_{i=1}^{m} p_{i}(s) \Delta s \leq 0
$$

or

$$
x(\sigma(t))+x(h(t))\left[\int_{h(t)}^{\sigma(t)} \sum_{i=1}^{m} p_{i}(s) \Delta s-1\right] \leq 0 .
$$

Consequently,

$$
\int_{h(t)}^{\sigma(t)} \sum_{i=1}^{m} p_{i}(s) \Delta s \leq 1-\frac{x(\sigma(t))}{x(h(t))}
$$

which gives

$$
\limsup _{t \rightarrow \infty} \int_{h(t)}^{\sigma(t)} \sum_{i=1}^{m} p_{i}(s) \Delta s \leq 1-\liminf _{t \rightarrow \infty} \frac{x(\sigma(t))}{x(h(t))}
$$

and by $(2.4),(2.7)$ leads to

$$
\limsup _{t \rightarrow \infty} \int_{h(t)}^{\sigma(t)} \sum_{i=1}^{m} p_{i}(s) \Delta s \leq 1-\frac{1-\alpha-\sqrt{1-2 \alpha-\alpha^{2}}}{2},
$$

which contradicts with (2.5). The proof of the theorem is completed.

Example 2.6. Let $m=1, h \in \mathbb{Z}$ and $\mathbb{T}=h \mathbb{Z}=\{h k: k \in \mathbb{Z}\}$, where $h>0$. Then, we have

$$
\sigma(t)=t+h, \quad \mu(t)=h \text { and } x^{\Delta}(t)=\frac{x(t+h)-x(t)}{h}
$$

for $t \in \mathbb{T}$. Thus, Eq.(1.1) becomes

$$
\frac{x(t+h)-x(t)}{h}+p(t) x(\tau(t))=0, \quad t \in\{h k: k \in \mathbb{Z}\} .
$$

Let $\tau(t)=t-2$ and $h=2$. Since $p(t) \in\{h k: k \in \mathbb{Z}\}$, we assume

$$
p(2 t)=0.18 \text { and } p(2 t+2)=0.27, \quad t=0,2,4, \ldots
$$

When $\mathbb{T}=h \mathbb{Z}$, from (iii) in [2, Theorem 1.79], we have the following.

$$
\int_{a}^{b} f(t) \Delta t=\sum_{k=\frac{a}{h}}^{\frac{b}{h}-1} f(k h) h \text { for } a<b .
$$

So, by using (2.8), we observe that, for $\tau(t), p(t) \in\{h k: k \in \mathbb{Z}\}$.

$$
\alpha:=\liminf _{t \rightarrow \infty} \int_{\tau(t)}^{t} p(s) \Delta s=\liminf _{t \rightarrow \infty} \sum_{j=\frac{t-2}{2}}^{\frac{t}{2}-1} p(2 j) 2=\liminf _{t \rightarrow \infty} 2 p(t-2)=0.36 \ngtr \frac{1}{e}
$$


and

$$
\beta:=\limsup _{t \rightarrow \infty} \int_{h(t)}^{\sigma(t)} p(s) \Delta s=\limsup _{t \rightarrow \infty} \sum_{j=\frac{t-2}{2}}^{\frac{t+2}{2}-1} p(2 j) 2=\limsup _{t \rightarrow \infty} 2[p(t-2)+p(t)]=0.9 \ngtr 1
$$

shows that Theorem 2.3 fails. On the other hand,

$$
\beta=0.9 \ngtr 1-(1-\sqrt{1-0.36})^{2}=0.96
$$

demonstrates that the condition (1.8) doesn't hold. However, since

$$
\beta=0.9>1-\frac{1-0.36-\sqrt{1-2(0.36)-(0.36)^{2}}}{2}=0.87391,
$$

every solution oscillates by Theorem 2.5 .

\section{References}

[1] R.P. Agarwal and M. Bohner, An oscillation criterion for first order delay dynamic equations, Funct. Differ. Equ. 16 (1), 11-17, 2009.

[2] M. Bohner and A. Peterson, Dynamic Equations on Time Scales: An Introduction with Applications, Birkhauser, Boston, 2001.

[3] M. Bohner and A. Peterson, Advances in Dynamic Equations on Time Scales, Birkhauser, Boston, 2003.

[4] M. Bohner, Some oscillation criteria for first order delay dynamic equations, Far East J. Appl. Math. 18 (3), 289-304, 2005.

[5] G.E. Chatzarakis, R. Koplatadze and I.P. Stavroulakis, Oscillation criteria of first order linear difference equations with delay argument, Nonlinear Anal. 68, 994-1005, 2008.

[6] G.E. Chatzarakis, R. Koplatadze and I.P. Stavroulakis, Optimal oscillation criteria for first order difference equations with delay argument, Pacific J. Math. 235, 15-33, 2008.

[7] G.E. Chatzarakis, Ch.G. Philos and I.P. Stavroulakis, On the oscillation of the solutions to linear difference equations with variable delay, Electron. J. Differ. Equ. 2008 (50), 1-15, 2008.

[8] G.E. Chatzarakis, Ch.G. Philos and I. P. Stavroulakis, An oscillation criterion for linear difference equations with general delay argument, Port. Math. 66 (4), 513-533, 2009.

[9] A. Elbert and I.P. Stavroulakis, Oscillations of first order differential equations with deviating arguments, Univ of Ioannina TR No 172, 1990, Recent trends in differential equations, 163-178, World Sci. Ser. Appl. Anal., 1, World Sci. Publishing Co., 1992.

[10] L.H. Erbe and B.G. Zhang, Oscillation of first order linear differential equations with deviating arguments, Differential Integral Equations 1, 305-314, 1988.

[11] L.H. Erbe and B.G. Zhang, Oscillation of discrete analogues of delay equations, Differential Integral Equations 2, 300-309, 1989.

[12] L.H. Erbe, Qingkai Kong and B.G. Zhang, Oscillation Theory for Functional Differential Equations, Marcel Dekker, New York, 1995.

[13] N. Fukagai and T. Kusano, Oscillation theory of first order functional differential equations with deviating arguments, Ann. Mat. Pura Appl. 136, 95-117, 1984.

[14] I. Györi and G. Ladas, Oscillation Theory of Delay Differential Equations with Applications, Clarendon Press, Oxford, 1991.

[15] S. Hilger, Ein MaXkettenkalkWul mit Anwendung auf Zentrumsmannigfaltigkeiten, Ph.D. thesis, Universtat Wurzburg, 1988.

[16] S. Hilger, Analysis on measure chainsa unified approach to continuous and discrete calculus, Results in Mathematics 18, 1856, 1990. 
[17] J. Jaroš and I.P. Stavroulakis, Oscillation tests for delay equations, Rocky Mountain J. Math. 29, 139-145, 1999.

[18] C. Jian, Oscillation of linear differential equations with deviating argument, Math. Pract. Theor. 1, 32-41, 1991 (in Chinese).

[19] B. Karpuz and Ö. Öcalan, New oscillation tests and some refinements for first-order delay dynamic equations, Turkish J. Math. 40 (4), 850-863, 2016.

[20] B. Karpuz, Sharp oscillation and nonoscillation tests for linear difference equations, J. Difference Equ Appl. 23 (12), 1929-1942, 2017.

[21] R.G. Koplatadze and T.A. Chanturija, Oscillating and monotone solutions of firstorder differential equations with deviating arguments, (Russian), Differentsial'nye Uravneniya 8, 1463-1465, 1982.

[22] M.K. Kwong, Oscillation of first-order delay equations, J. Math. Anal. Appl. 156, 274-286, 1991.

[23] G.S. Ladde, V. Lakshmikantham and B.G. Zhang, Oscillation theory of differential equations with deviating arguments, Monographs and Textbooks in Pure and Applied Mathematics, vol. 110, Marcel Dekker, Inc., New York, 1987.

[24] Ö. Öcalan, U.M. Özkan and M.K. Ylldı, Oscillatory solutions for dynamic equations with non-monotone arguments, J. Math. Comput. Sci. 7 (4), 725-738, 2017.

[25] Ö. Öcalan, Oscillation of first-order dynamic equations with nonmonotone delay, Math. Methods Appl. Sci. 43 (7), 3954-3964, 2020.

[26] Ch.G. Philos and Y.G. Sficas, An oscillation criterion for first-order linear delay differential equations, Canad. Math. Bull. 41, 207-213, 1998.

[27] Y. Şahiner and I.P. Stavroulakis, Oscillations of first order delay dynamic equations, Dynam. Systems Appl. 15 (3-4), 645-655, 2006.

[28] J.S. Yu and Z.C. Wang, Some further results on oscillation of neutral differential equations, Bull. Aust. Math. Soc. 46, 149-157, 1992.

[29] J.S. Yu, Z.C. Wang, B.G. Zhang and X.Z. Qian, Oscillations of differential equations with deviating arguments, PanAmerican Math. J. 2, 59-78, 1992.

[30] B.G. Zhang and C.J. Tian, Oscillation criteria for difference equations with unbounded delay, Comput. Math. Appl. 35 (4), 19-26, 1998.

[31] B.G. Zhang and C.J. Tian, Nonexistence and existence of positive solutions for difference equations with unbounded delay, Comput. Math. Appl. 36, 1-8, 1998.

[32] B.G. Zhang and X. Deng, Oscillation of delay differential equations on time scales, Math. Comput. Modelling 36 (11-13), 1307-1318, 2002.

[33] B.G. Zhang, X. Yan and X. Liu, Oscillation criteria of certain delay dynamic equations on time scales, J. Difference Equ. Appl. 11 (10), 933-946, 2005.

[34] Y. Zhou and Y.H. Yu, On the oscillation of solutions of first order differential equations with deviating arguments, Acta Math. Appl. Sinica 15 (3), 288-302, 1999. 